Research Journal of Applied Sciences 7 (6): 316-321, 2012

ISSN: $1815-932 \mathrm{X}$

(C) Medwell Journals, 2012

\title{
Mismatch Negativity of the Contour Tone Changes in Monosyllabic Thai Words
}

\author{
Wichian Sittiprapaporn \\ Faculty of Medicine, Mahasarakham University, 44000 Maha Sarakham, Thailand
}

\begin{abstract}
As both hemispheres are lateralized for speech and language, the objective of this study is to extend the investigation of how the preattentive processing of contour tone changes in Thai monosyllabic words. Twenty two healthy right handed adults participated in this study. This study found that both rising to falling and falling to rising tone changes perception elicited $\mathrm{MMN}$ between $212-244 \mathrm{msec}$ with reference to the standard-stimulus ERPs. The rising to falling and falling to rising tone changes elicited a strong MMN bilaterally for native and nonnative speakers of Thai. Source localization was obtained in the Middle Temporal Gyrus (MTG) of the left hemisphere and in the Superior Temporal Gyrus (STG) of the right hemisphere for both groups. Automatic detection of changes in contour tones is a useful index of language universal auditory memory traces.
\end{abstract}

Key words: Event-related potential, sound, tone, contour tone, mismatch negativity, Thailand

\section{INTRODUCTION}

In earlier studies (Gandour, 1998; Gandour et al., 2000; Hsieh et al., 2001), Chinese and English listeners did not show the same Left-Hemisphere (LH) lateralization as Thai listeners when making perceptual judgments of Thai tones. In addition, Chinese and English listeners were asked to make perceptual judgments of Chinese tones, consonants and vowels. Chinese listeners showed LeftHemisphere (LH) lateralization for both suprasegmental and segmental phonological units (Hsieh et al., 2001). These earlier studies suggest that functional circuits engage in early, pre-attentive speech perception of either suprasegmental or segmental units in tone languages. While it seems indisputable that language is subserved by Left-Hemisphere $(\mathrm{LH})$ and Right-Hemisphere $(\mathrm{RH})$ are lateralized for speech, language or something else. Hypotheses proposed to account for functional hemispheric asymmetries can generally be classified as either cue dependent, i.e., basic neural mechanism underlie processing of complex auditory stimuli regardless of linguistic relevance (Ivry and Roberson, 1998) or task dependent, i.e., specialized neural mechanisms exist that are activated only by speech (Imaizumi et al., 1998).

Previous ERP studies at a phonetic level demonstrated that the MMN was enhanced in Finnish subjects by their first-language (Finnish) phoneme prototype rather than a non-prototype (Estonian) (Naatanen, 1992) and that the MMN for a vowel contrast in Finnish was not generated in native Hungarian speakers with no knowledge of Finnish (Winkler et al., 1999), implying that the $M M N$ reflects language-specific memory traces formed by early and extensive exposure to a first language. However, language-specific word-related
MMN/MMF components at acoustic and phonetic levels remain to be investigated in future studies. The differences between these studies provide the impetus for future investigations of duration processing and temporal integration differences across language groups. Although, the left hemisphere is selectively employed for processing linguistic information irrespectively of acoustic cues or subtype of phonological unit, the right hemisphere is employed for prosody-specific cues (Imaizumi et al., 1998). The propose of the present study is thus to use both an auditory MMN component of Event-Related Potential (ERP) recording and the Low Resolution Electromagnetic Tomography (LORETA) techniques to measure the degree of cortical activation and to localize the brain area contributing to the scalp recorded auditory $\mathrm{MMN}$ component, respectively during the passive oddball paradigm. Thus, the objective of this study is to investigate the preattentive processing of contour tone changes in Thai monosyllabic words.

\section{MATERIALS AND METHODS}

Subjects: Twenty two healthy right-handed adults with normal hearing and no known neurological disorders volunteered for participation: eleven Native Speakers (NS), aged 23-39 (mean 25.3; six females) and eleven Non-native Speakers (NonS), aged 23-29 (mean 27.8; nine females). The approval of the institutional committee on human research and written consent from each subject were obtained.

Stimuli and procedure: Stimuli consisted of two pairs of monosyllabic, Thai words. Speech stimuli were digitally generated and edited to have equal peak energy level in 
decibels SPL with the remaining data within each of the stimuli scaled accordingly using the Cool Edit Pro v. 2.0 (Syntrillium Software Corporation). The sound pressure levels of speech stimuli were then measured at the output of the earphones (E-A-RTONE 3A, 50U) in dBA using a Bruel and Kjaer 2230 sound-level meter. Pairs were designed to have similar long vowel duration. Two different stimuli were synthetically generated as follows:

- Stimuli $1: / \mathrm{K}^{\mathrm{h}}$ aam/- falling tone

- Stimuli $2: / \mathrm{K}^{\mathrm{h}}$ aam/- rising tone

Five NS listened to the synthesized words and evaluated them all as natural sounding. The Standard (S)/Deviant (D) pairs for each experiment which was randomized across subjects were shown as follows:

- [Experiment 1] Standard (1) Deviant

- [Experiment 2] Standard (1) Deviant

The sounds were presented binaurally via headphones (Telephonic TDH-39-P) at $85 \mathrm{~dB}$. The Inter-Stimulus Interval (ISI) was $1.25 \mathrm{sec}$ (offset-onset). Deviant stimuli appeared randomly among the standards at $10 \%$ probability. Each experiment included 125 trials $(10 \%$ D). The stimuli were binaurally delivered using SuperLab Software (Cedrus Corporation, San Pedro, USA) via headphones (Telephonic TDH-39-P). EEG signal recording was time-locked to the onset of a word. Subjects were instructed not to pay attention to the stimuli presented via headphones but rather to concentrate on a self-selected silent, subtitled movie.

Electroencephalographic recording: For EEG/ERP recording, the standard 20 locations of the 10-20 system, EEG is recorded via an electrocap (Electrocap International) from 20 active electrodes (Fpl, Fp2, F7, F3, Fz, F4, T3, C3, Cz, C4, T4, T5, P3, Pz, P4, T6, O1, Oz, O2) positioned according to the 10-20 International System of Electrode Placement, plus $\mathrm{Oz}$ and Ground are applied, pre-mounted in an elastic Electro-Cap. Reference electrodes are manually applied to left and right mandibles where the Fp1 and Fp2 electrodes are used for ocular artifact detection. Horizontal eye movements are monitored with electrodes at the left and right outer canthi and vertical eye movements are monitored at $\mathrm{Fp} 1$ and $\mathrm{Fp} 2$. EEG is amplified with a gain of 30,000 and filtered with a bandpass of 0.1-30 Hz. EEGs are acquired as continuous signals and are subsequently segmented into epochs of $1 \mathrm{sec}$ (a $100 \mathrm{msec}$ pre-stimulus baseline and a $900 \mathrm{msec}$ post-stimulus epoch).
EEG data processing: The recordings were filtered and carefully inspected for eye movement and muscle artifacts. ERPs were obtained by averaging epoch which started $100 \mathrm{msec}$ before the stimulus onset and ended $900 \mathrm{msec}$ thereafter the -100 to $0 \mathrm{msec}$ interval was used as a baseline. Epochs with voltage variation exceeding $100 \mathrm{FV}$ at any EEG channel were rejected from further analysis. The $M M N$ was obtained by subtracting the response to the standard from that to the deviant stimulus. All responses were recalculated offline against average reference for further analysis.

Spatial analysis: The average $M M N$ latency was defined as a moment of the global field power with an epoch of $40 \mathrm{msec}$ time window related stable scalp-potential topography (Pascual-Marqui et al., 1994). In the next step, Low-Resolution Electromagnetic Tomography (LORETA) is applied to estimate the current source density distribution in the brain which contributes to the electrical scalp field (Pascual-Marqui et al., 1994). Maps are computed with LORETA. LORETA computes the smoothest of all possible source configurations throughout the brain volume by minimizing the total squared Laplacian of source strengths.

Data analysis: During the auditory stimulation, electric activity of the subjects brain was continuously recorded. The MMN was obtained by subtracting the response to the standard from that to the deviant stimulus. The statistical significance of $\mathrm{MMN}$ was tested with one sample t-test. An across-experiment ANOVA was carried out so as to make cross-linguistic comparisons.

\section{RESULTS AND DISCUSSION}

The grand-averaged ERPs show that both rising to falling and falling to rising tone changes perception elicited MMN between 212-244 msec with reference to the standard-stimulus ERPs. An ANOVA comparing MMN amplitudes of the $\mathrm{S}$ and $\mathrm{D}$ were not significant $\left(\mathrm{F}_{(3,40)}=0.62, \mathrm{p}=0.55\right.$ in Experiment $1, \mathrm{NS}$ and $\mathrm{F}_{(3,40)}=0.23$, $\mathrm{p}=0.82$ in Experiment 2, NS for the main effect of conditions). The result showed that rising to falling and falling to rising tone changes elicited a strong $\mathrm{MMN}$ bilaterally for NS and NonS (Table 1). Furthermore, an across-experiment ANOVA demonstrated no interaction and main effects. The significant difference in $M M N$ amplitudes was not observed between groups across experiments $\left(\mathrm{F}_{(7,80)}=0.12, \mathrm{p}=0.9555, \mathrm{NS}\right)$.

Source localization analyses were performed using LORETA-Key (Pascual-Marqui et al., 1994). Table 2 demonstrates the xyz-values in Talairach space as 
calculated with LORETA in the time window 212-224 msec. In Experiment 2, a single source was estimated to be located in the Middle Temporal Gyrus (MTG) of each hemisphere for both groups. In

Table 1: Mean amplitude $(\mu \mathrm{V}) \pm \mathrm{SD}$ of MMN elicited by a contour tone change perception in NS and NonS

\begin{tabular}{lcc}
\hline Tone changes & $\mathrm{NS}$ & NonS \\
\hline Rising to falling & $-3.03 \pm 0.87$ & $-3.13 \pm 1.08$ \\
Falling to rising & $-2.92 \pm 0.95$ & $-2.99 \pm 0.70$ \\
\hline
\end{tabular}

Table 2: Stereotaxic coordinates of activation foci during the contour tones change perception

\begin{tabular}{|c|c|c|c|c|c|}
\hline \multirow{2}{*}{ Tone changes } & \multicolumn{5}{|c|}{ Coordinates (mm) } \\
\hline & $\mathrm{x}$ & $\mathrm{y}$ & $z$ & $\mathrm{BA}$ & t-values \\
\hline \multicolumn{6}{|c|}{ Native Speakers (NS) } \\
\hline \multirow[t]{2}{*}{ Rising to falling } & 39 & 45 & -67 & $15^{\mathrm{a}}$ & 5.91 \\
\hline & 22 & 53 & -60 & $15^{b}$ & 4.69 \\
\hline \multirow[t]{2}{*}{ Falling to rising } & 21 & -59 & -32 & $1^{\mathrm{a}}$ & 5.73 \\
\hline & 37 & 46 & -67 & $8^{c}$ & 4.64 \\
\hline \multicolumn{6}{|c|}{ Nonnative Speaker (NonS) } \\
\hline \multirow[t]{2}{*}{ Rising to falling } & 39 & -52 & -67 & 15 & 1.82 \\
\hline & 22 & 53 & -60 & $15^{b}$ & 1.12 \\
\hline \multirow[t]{2}{*}{ Falling to rising } & 39 & -45 & -67 & $15^{\mathrm{a}}$ & 1.10 \\
\hline & 37 & 46 & -67 & $8^{c}$ & 1.06 \\
\hline
\end{tabular}

${ }^{a}$ L Middle Temporal Gyrus (MTG); R Superior Temporal Gyrus (STG): ${ }^{\mathrm{c}} \mathrm{R}$ Middle Temporal Gyrus (MTG)
Experiment 1, sources were obtained in the MTG of the $\mathrm{LH}$ and in the Superior Temporal Gyrus (STG) of the $\mathrm{RH}$ for both groups. No hemispheric difference was discovered in this study (Table 2 and Fig. 1-4).

Both rising to falling and falling to rising tone changes perception elicited MMN between 212-244 msec with reference to the standard-stimulus ERPs. The rising to falling and falling to rising tone changes elicited a strong $\mathrm{MMN}$ bilaterally for native and nonnative speakers of Thai. Source localization analyses performed using LORETA-Key demonstrates that sources were obtained in the Middle Temporal Gyrus (MTG) of the left hemisphere and in the Superior Temporal Gyrus (STG) of the right hemisphere for both groups. The same results were obtained in MEG studies using both tone and Japanese words (Inouchi et al., 2002, 2003). The present results parallel the finding in previous studies (Inouchi et al., 2002, 2003). The present study, the detection of vowel duration and tone changes was most likely acoustically driven rather than semantically driven such that the stimuli were processed without any access to semantic information. The acoustic aspect in the
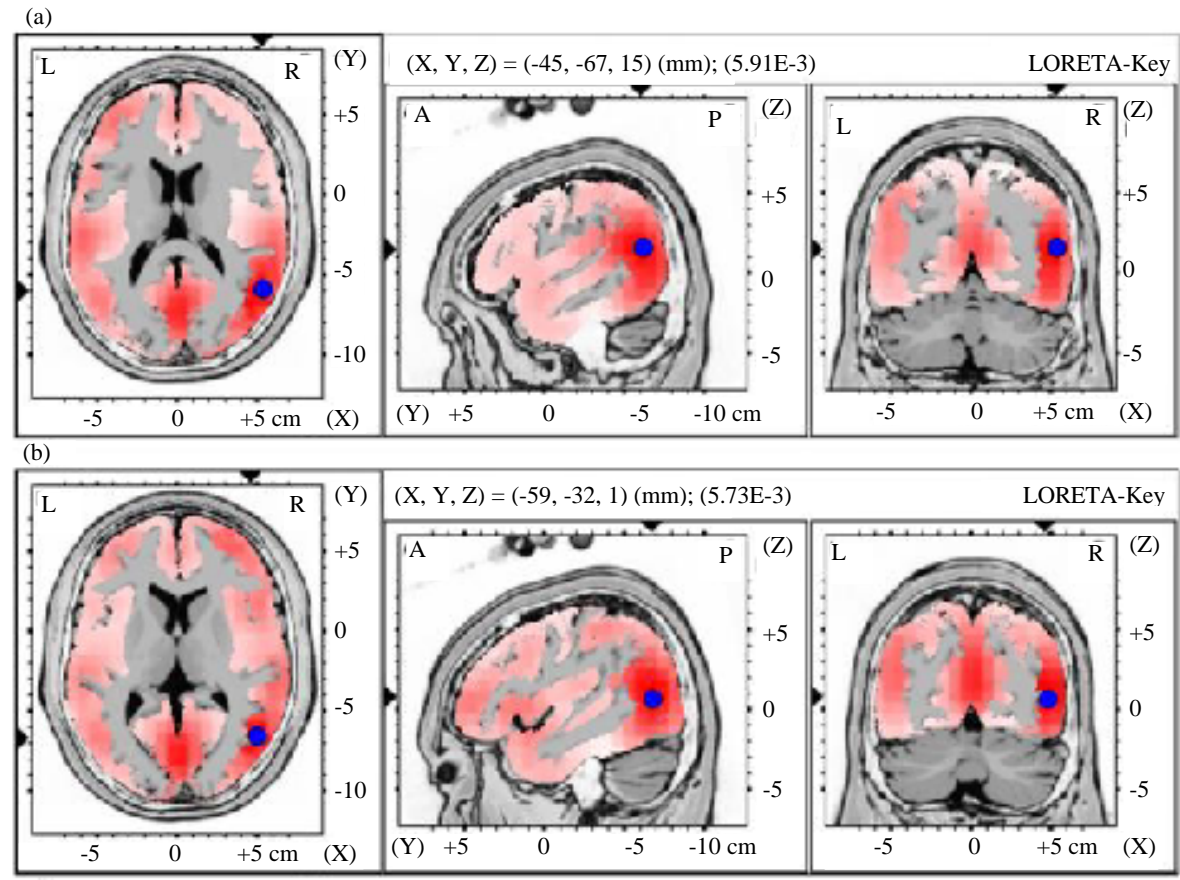

Fig. 1: Graphical representation of the Low-Resolution Electromagnetic Tomography (LORETA t-statistic comparing the event-related potentials for Mismatch Negativity (MMN) responses at the time point of the individual peak over $\mathrm{Fz}$ for; $\mathrm{a}$ ) the rising to falling and $\mathrm{b}$ ) the falling to rising tone changes occupied by the long vowel duration of Native Speaker (NS) activated in Left Hemisphere (LH). Red color indicates local maxima of increased electrical activity for across and within category of vowel change responses in an axial, a saggital and a coronal slice through the reference brain. Blue dots mark the center of significantly increased electric activity 

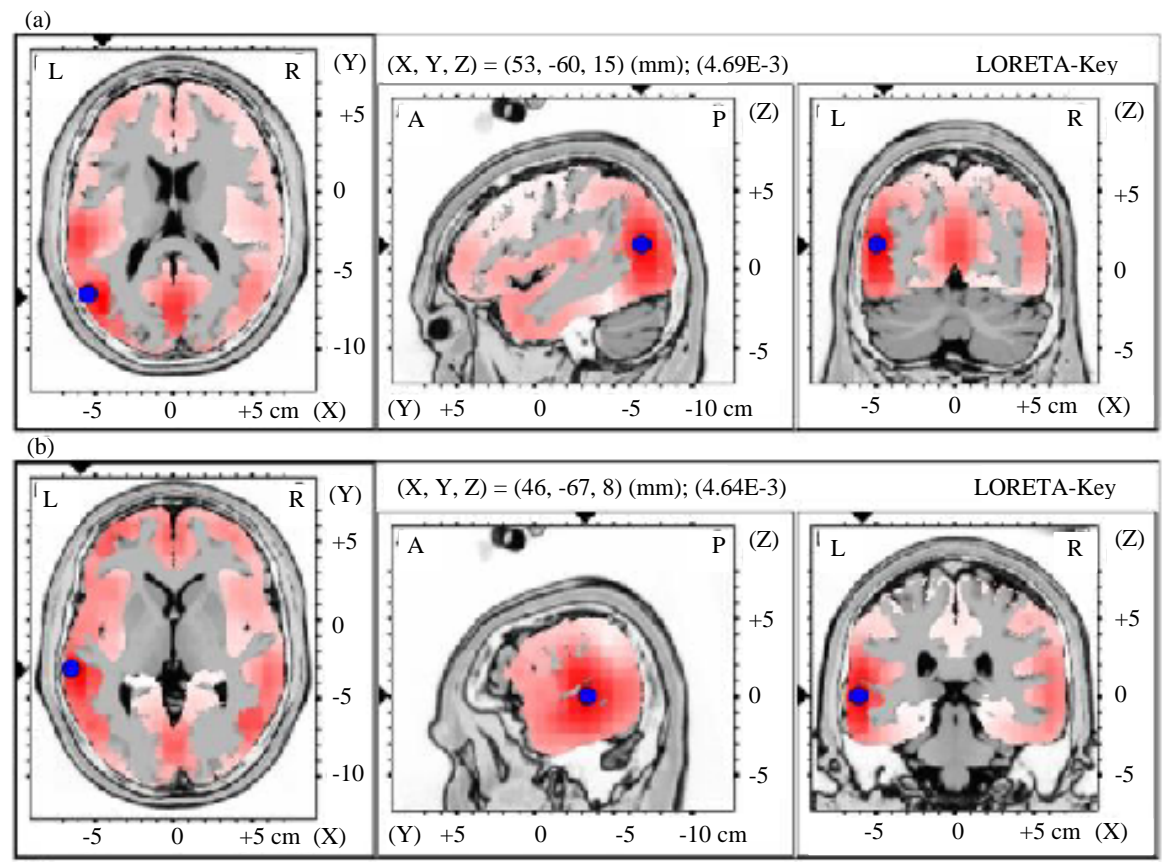

Fig. 2: Graphical representation of the Low-Resolution Electromagnetic Tomography (LORETA t-statistic comparing the event-related potentials for $\mathrm{MMN}$ responses at the time point of the individual peak over $\mathrm{Fz}$ for; a) the rising to falling and $\mathrm{b}$ ) the falling to rising tone changes occupied by the long vowel duration of NS activated in $\mathrm{RH}$
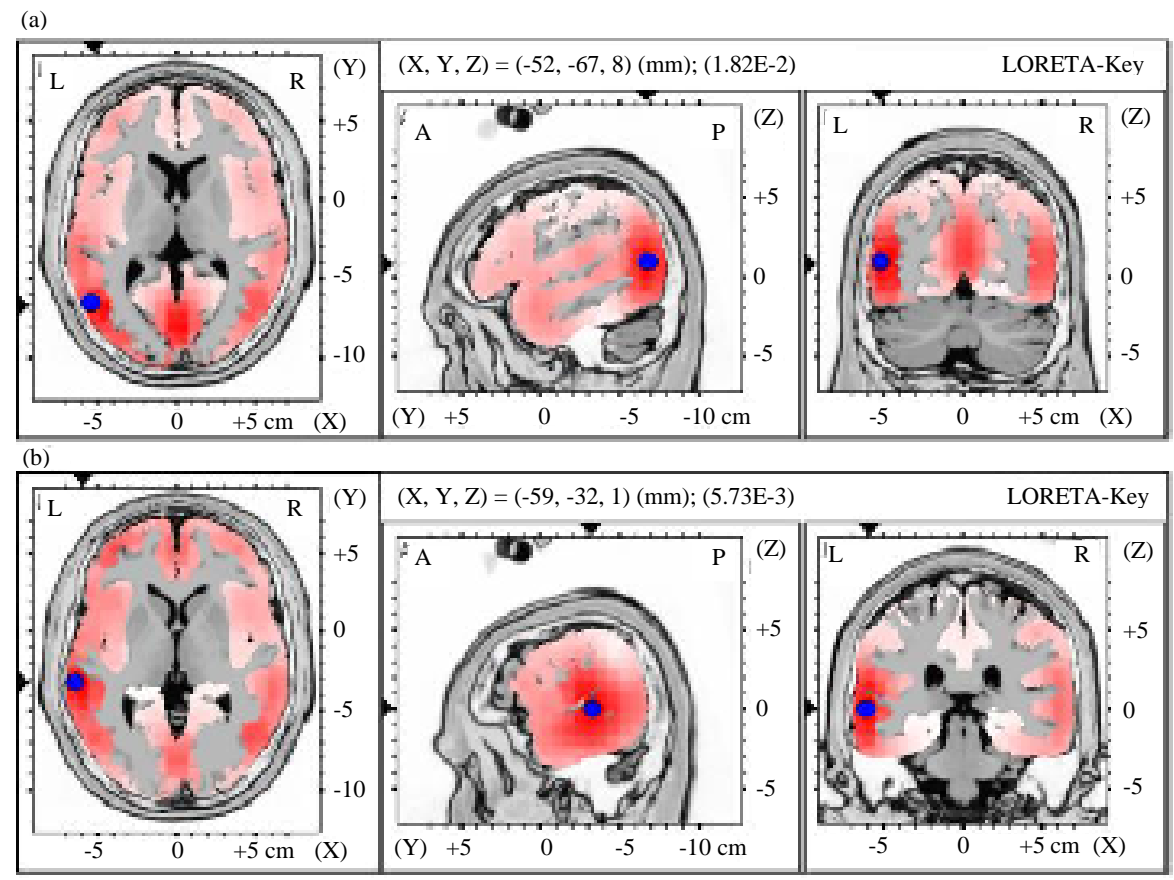

Fig. 3: Graphical representation of the Low-Resolution Electromagnetic Tomography (LORETA t-statistic comparing the event-related potentials for MMN responses at the time point of the individual peak over Fz for; a) the rising to falling and $\mathrm{b}$ ) the falling to rising tone changes occupied by the long vowel duration of NonS activated in $\mathrm{LH}$ 

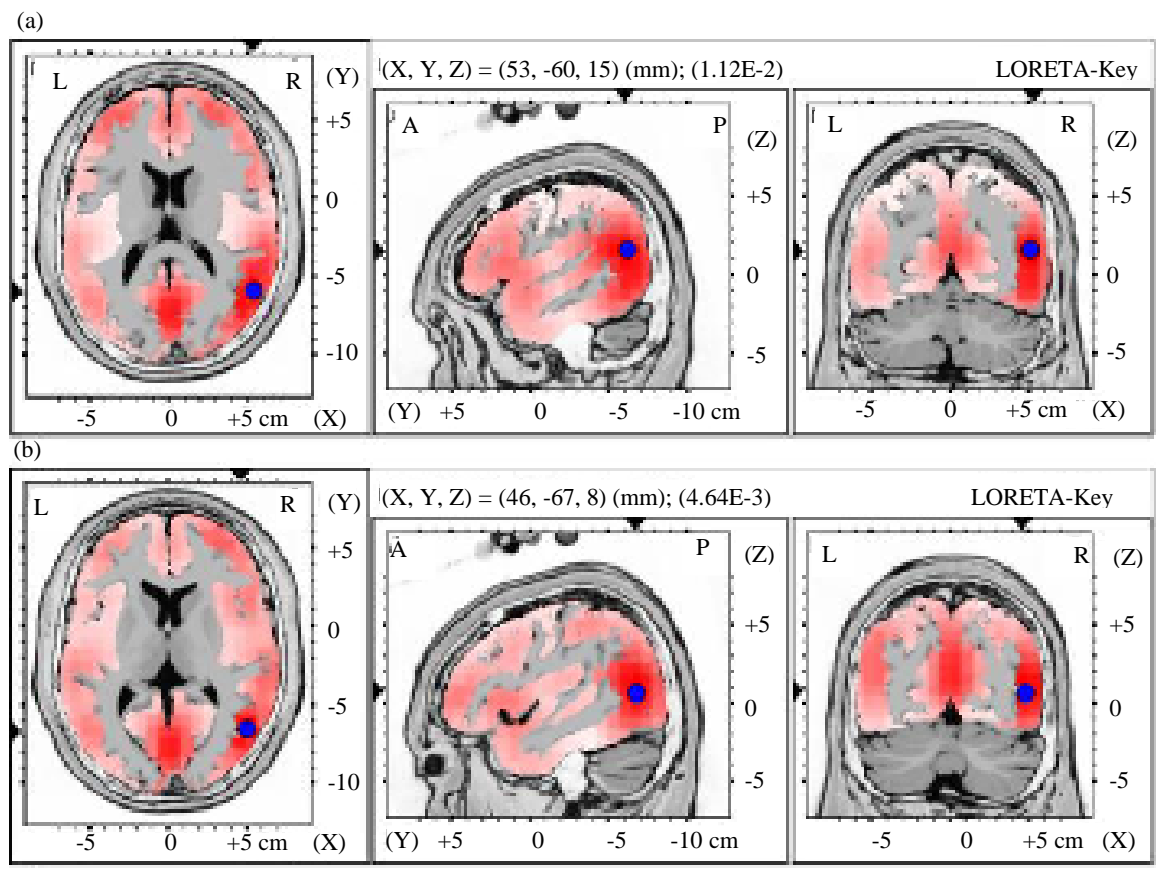

Fig. 4: Graphical representation of the Low-Resolution Electromagnetic Tomography (LORETA t-statistic comparing the event-related potentials for $\mathrm{MMN}$ responses at the time point of the individual peak over $\mathrm{Fz}$ for; a) the rising to falling and $\mathrm{b}$ ) the falling to rising tone changes occupied by the long vowel duration of NonS activated in $\mathrm{RH}$

absence of phonetic or higher-order properties may account for why NonS had similar neuronal responses to NS subjects. This suggests that at the point of stimulus disparity or thereafter, change detection of vowel lengthening is somehow compromised. It is reasonable to speculate that the continued auditory processing required for the longer vowel interferes with or masks the detection mechanism underlying the MMN (Inouchi et al., 2002). In contrast, the current findings contradict previous tone studies that reported a clear $\mathrm{MMN}$ elicited by both duration increments and decrements (Naatanen et al., 1989) and a larger MMN elicited by increments than decrements (Jaramillo et al., 1990). The MMN component was also found to be more sensitive to tone falling than rising and leveling for both groups.

One might expect language-specific effects on the elicitation of the MMN in speech since, Thai is a tonal language and English is a stress-accent language. Although, a tendency towards stronger MMN in NonS is observed, the current findings do not support such an expectation in that no statistically significant difference in MMN amplitudes was found between groups at an acoustic level. Previous ERP studies at a phonetic level demonstrated that the MMN was enhanced in Finnish subjects by their first-language (Finnish) phoneme prototype rather than a non-prototype (Estonian) (Gandour, 1998) and that the MMN for a vowel contrast in Finnish was not generated in native Hungarian speakers with no knowledge of Finnish (Winkler et al., 1999), implying that the MMN reflects language-specific memory traces formed by early and extensive exposure to a first language. However, language-specific word-related MMN/MMF components at acoustic and phonetic levels remain to be investigated in future studies.

\section{CONCLUSION}

Both rising to falling and falling to rising tone changes perception elicited MMN between 212-244 msec with reference to the standard-stimulus ERPs. The rising to falling and falling to rising tone changes elicited a strong MMN bilaterally for native and nonnative speakers of Thai. Source localization analyses performed using LORETA-Key demonstrates that sources were obtained in the Middle Temporal Gyrus (MTG) of the left hemisphere and in the Superior Temporal Gyrus (STG) of the right hemisphere for both groups. Automatic detection of changes in vowel shortening and pitch falling is a useful index of language universal auditory memory traces. 


\section{REFERENCES}

Gandour, J., 1998. Aphasia in Tone Languages. In: Aphasia in Atypical Populations, Coppens, P., A. Basso and Y. Lebrun (Eds.). Lawrence Erlbaum, Hillsdale, NJ., USA., pp: 117-141.

Gandour, J., D. Wong, L. Hsieh, B. Weinzapfel, D. van Lacker and G.D. Hutchins, 2000. A crosslinguistic PET study of tone perception. J. Cognitive Neurosci., 12: $207-222$.

Hsieh, L., J. Gandour, D. Wong and G.D. Hutchins, 2001. Functional heterogeneity of inferior frontal gyrus is shaped by linguistic experience. Brain Lang., 76: $227-252$.

Imaizumi, S., K. Mori, S. Kiritani, H. Hosoi and M. Tonoike, 1998. Task-dependent laterality for cue decoding during spoken language processing. Neuroreport, 9: 899-903.

Inouchi, M., M. Kubota, P. Ferrari and T.P.L. Roberts, 2002. Neuromagnetic auditory cortex responses to duration and pitch changes in tones: Cross-linguistic comparisons of human subjects in directions of acoustic changes. Neurosci. Lett., 331: 138-142.
Inouchi, M., M. Kubota, P. Ferrari and T.P.L. Roberts, 2003. Magnetic mismatch fields elicited by vowel duration and pitch changes in Japanese words in humans: Comparison between native-and nonspeakers of Japanese. Neurosci. Lett., 353: 165-168.

Ivry, R. and L. Roberson, 1998. The Two Sides of Perception. MIT Press, Cambridge, MA., USA.

Jaramillo, M., P. Alku and P. Paavilainen, 1990. An Event-Related Potential (ERP) study of duration changes in speech and non-speech sounds. Neuroreport, 10: 3301-3305.

Naatanen, R., 1992. Attention and Brain Function. Lawrence Erlbaurn, Hillsdale, USA.

Naatanen, R., P. Paavilainen and K. Reinikainen, 1989. Do event-related potentials to infrequent decrements in duration of auditory stimuli demonstrate a memory trace in man? Neurosci. Lett., 107: 347-352.

Pascual-Marqui, R.D., C.M. Michel and D. Lehmann, 1994. Low resolution electromagnetic tomography: A new method for localizing electrical activity in the brain. Int. J. Psychophysiol., 18: 49-65.

Winkler, I., A. Lehtokoski, P. Alku, M. Vainio and I. Czigler et al., 1999. Pre-attentive detection of vowel contrasts utilizes both phonetic and auditory memory representations. Cognitive Brain Res., 7: 357-369. 